\title{
On Randic, Seidel, and Laplacian Energy of NEPS Graph
}

\author{
Kun Han, ${ }^{1}$ S. Ahmad, ${ }^{2}$ Syed Ajaz K. Kirmani, ${ }^{3}$ M. K. Siddiqui, ${ }^{2}$ Y. Ali, ${ }^{2}$ and E. Bashier $\mathbb{D}^{4}$ \\ ${ }^{1}$ School of Management, Wuhan Polytechnic University, Wuhan, China \\ ${ }^{2}$ Department of Mathematics, COMSATS University Islamabad, Lahore Campus, Pakistan \\ ${ }^{3}$ Department of Electrical Engineering, College of Engineering, Qassim University, Unaizah, Saudi Arabia \\ ${ }^{4}$ Department of Applied Mathematics, Faculty of Mathematical Sciences, University of Khartoum, Sudan \\ Correspondence should be addressed to E. Bashier; eihabbashier@gmail.com
}

Received 9 January 2022; Accepted 8 February 2022; Published 28 February 2022

Academic Editor: Gul Rahmat

Copyright (c) 2022 Kun Han et al. This is an open access article distributed under the Creative Commons Attribution License, which permits unrestricted use, distribution, and reproduction in any medium, provided the original work is properly cited.

Let $Z$ be the simple graph; then, we can obtain the energy $E(Z)$ of a graph $Z$ by taking the absolute sum of the eigenvalues of the adjacency matrix of $Z$. In this research, we have computed different energy invariants of the noncompleted extended P-Sum (NEPS) of graph $Z_{i}$. In particular, we investigate the Randic, Seidel, and Laplacian energies of the NEPS of path graph $P_{n_{i}}$ with any base $\mathscr{B}$. Here, $n$ denotes the number of vertices and $i$ denotes the number of copies of path graph $P_{n}$. Some of the results depend on the number of zeroes in base elements, for which we use the notation $j$.

\section{Introduction}

The NEPS of the graphs is a graph $Z$ whose vertex set is equal to the simple Cartesian product of the vertices' sets of the graphs [1]. If $Z_{1}, Z_{2}, Z_{3}, \ldots, Z_{K}$ are $\mathrm{K}$ graphs having $v\left(Z_{1}\right), v\left(Z_{2}\right), v\left(Z_{3}\right), \ldots, v\left(Z_{K}\right)$ vertices sets, respectively, then the vertex set of NEPS of graphs is defined as

$$
V(Z)=v\left(Z_{1}\right) \times v\left(Z_{2}\right) \times v\left(Z_{3}\right) \times \cdots \times v\left(Z_{K}\right) .
$$

The existence of the edges of NEPS graph is depending on the base elements:

$$
\left(\beta_{1}, \beta_{2}, \beta_{3}, \ldots, \beta_{K}\right) \in \overline{\mathscr{B}}
$$

whereas some $\beta_{i}=0$ and some $\beta_{i}=1$. When $\beta_{i}=0$, it means there is no edge because in this case $u_{i}=v_{i}$. However, when $\beta_{i}=1$, then there exists an edge between $\left(u_{1}, u_{2}, \ldots, u_{K}\right)$ and $\left(v_{1}, v_{2}, \ldots, v_{K}\right)$ iff $Z_{i}$ has an edge between $u_{i}$ and $v_{i}$.

The NEPS of the graph is abbreviated as noncomplete extended p-sum of the graph. It has many graph operations as special cases, and names are as follows: the product, the sum, and the strong product of graphs [1]. The energy of graph is the summation of $\left|\lambda_{i}\right|$ of adjacency matrix $A(G)$ of graph $G[2,3]$. Energy of graphs, first introduced by Ivan
Gutman, has remarkable chemical application; see [4-6], for details.

This energy have applications in image analysis $[7,8]$, which is been used in investigation of medical fields like in brain activity. Randic energy of graph $G$ is the summation of $\left|\rho_{i}\right|$, where $\rho_{i}$ are the eigenvalues of the Randic matrix $R(G)$ $[9,10]$. It has endless applications in chemistry and became an attractive field of research; for further details, see [11-13]. In [7], Haemers defined the Seidel energy; let the Seidel matrix of a graph $G$ be represented as $\operatorname{SE}(G)$, and $\theta_{i}$ are the eigenvalues of this matrix; then, the energy of graph is the summation of $\left|\theta_{i}\right|$, where $\theta-i$ is of the Seidel matrix. More results on this energy can be found in [14-16]. These energies have lot of work in calculating upper and lower bounds of different graphs. In Section 2, we first show NEPS of the cyclic graph and how the energy of NEPS with any other basis do not have any effect on energies, see [17-19]. Then, how this result is used in calculating Randic, Seidel, and Laplacian energy of this graph?

Example 1. Consider two graphs $P_{2}$ and $C_{3}$; then, the vertex set of NEPS of $P_{2}$ and $C_{3}$ is stated as

$$
V(Z)=V\left(P_{2}\right) \times V\left(C_{3}\right) .
$$


Let $V\left(P_{2}\right)=(1,2)$ and $V\left(C_{3}\right)=\left(1^{\prime}, 2^{\prime}, 3^{\prime}\right)$; then,

$V(Z)=\left\{\left(1,1^{\prime}\right),\left(1,2^{\prime}\right),\left(1,3^{\prime}\right),\left(2,1^{\prime}\right),\left(2,2^{\prime}\right),\left(2,3^{\prime}\right)\right\}$.

The NEPS graph is dependent on the base elements, and the possible base elements are $\{(1,1),(1,0),(0,1)\}$. If we take the base element $(1,1)$, then the graphical view of $\operatorname{NEPS}\left\{P_{2} \times C_{3}:(1,1)\right\}$ is expressed as in Figure 1 .

If we take base element $(0,1)$, then its graph is shown in Figure 2.

Now, for $(1,0)$, we have Figure 3 .

If we take three copies of $P_{2}$ and $(1,1,1)$ as the base element, then its NEPS graph is expressed as in Figure 4.

Now, for three copies of $P_{2}$, with base $(1,0,1)$, the NEPS graph is shown in Figure 5.

The NEPS graph was first time introduced by D. Cvetkovic and R. Lucic [3], and after some time, it was redefined by S.C. Shee [20]. It has defined various graph operations; some of the results we explain as follows. Let $Z_{1}$ and $Z_{2}$ be any two graphs; if we take bases $\{(1,0),(0,1)\}$, then the resulting graph $Z$ is the sum of $Z_{1}$ and $Z_{2}$; if the base element is $(1,1)$, then the graph $Z$ is the tensor product of $Z_{1}$ and $Z_{2}$. Furthermore, the connectedness of the NEPS graph in [2] and NEPS operation on cordial graphs in [8] have been discussed.

The energy of the graph $Z$ is defined as the absolute sum of the eigenvalues of the adjacency matrix of $Z$. It was first time introduced by Ivan Gutman [5]. The adjacency matrix of the simple graph $Z$ is a matrix whose entries are zeroes and ones. If the vertices $v_{i} v_{j} \in E$, then $A_{i j}=1$; otherwise, $A_{i j}=0$. It is denoted by $A(Z)$.

A Randic matrix $R(Z)$ is a matrix whose entries are denoted by $r_{i j}$ and is defined as $R=\left[r_{i j}\right]$. If $u_{i}$ and $u_{j}$ are not adjacent or $u_{i}=u_{j}$, then $r_{i j}=0$, but if $u_{i}$ and $u_{j}$ are adjacent, then $r_{i j}=1 / \sqrt{d_{i} d_{j}}$, where $d_{i}$ and $d_{j}$ are degrees of $u_{i}$ and $u_{j}$, respectively. The Randic energy of a graph $Z$ can be evaluated by taking the absolute sum of the eigenvalues of the Randic matrix. If the eigenvalues of the Randic matrix are denoted by $\mu_{i}$, then we can express the Randic energy of $Z$ as [21]

$$
R E(Z)=\sum_{i=1}^{n}\left|\mu_{i}\right| .
$$

Milan Randic is the first person who has introduced the Randic index [22] such as

$$
R(Z)=\sum_{i \sim j} \frac{1}{\sqrt{d_{i} d_{j}}} .
$$

Gutman et al. have explained that the Randic index is used to form the Randic matrix [5]. Theye also introduced the energy of the graphs $[9,10]$; then, they extended this topic and defined the Randic energy [15]. Dasa et al. have discussed the upper and lower bounds on the Randic energy of the graphs [4].

Let $Z$ be a graph having vertices $\left\{v_{1}, v_{2}, v_{3}, \ldots, v_{n}\right\}$; then, the Seidel matrix of $Z$ is a $n \times n$ matrix including the entries $-1,0$, and 1 . The Seidel matrix is expressed as $S(Z)=\left[s_{i j}\right]$. If

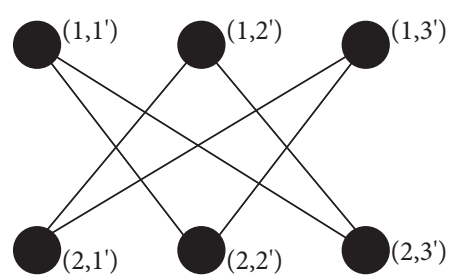

Figure 1: $\operatorname{NEPS}\left\{P_{2} \times C_{3}:(1,1)\right\}$.
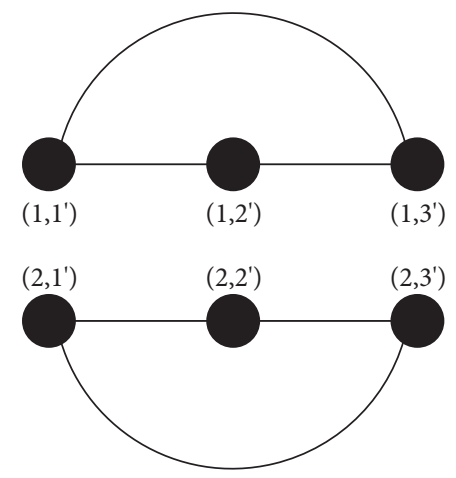

Figure 2: NEPS $\left\{P_{2} \times C_{3}:(0,1)\right\}$.

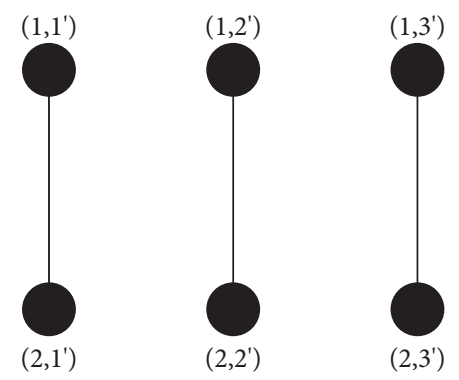

Figure 3: NEPS $\left\{P_{2} \times C_{3}:(1,0)\right\}$.

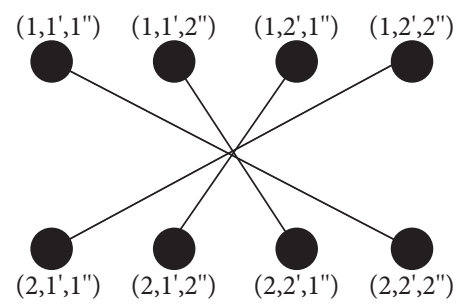

Figure 4: $\operatorname{NEPS}\left\{P_{2} \times P_{2} \times P_{2}:(1,1,1)\right\}$.

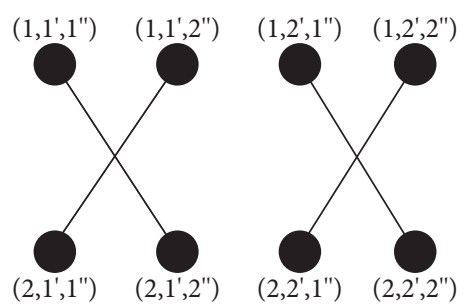

Figure 5: NEPS $\left\{P_{2} \times P_{2} \times P_{2}:(1,0,1)\right\}$. 
$v_{i}=v_{j}$, then $s_{i j}=0$; if $v_{i} \neq v_{j}$ and there exist an edge between $v_{i}$ and $v_{j}$ in $Z$, then $s_{i j}=-1$, but if $v_{i} \neq v_{j}$ and there does not exist an edge between $v_{i}$ and $v_{j}$ in $Z$, then $s_{i j}=1$. Let the eigenvalues of the Seidel matrix be denoted by $\theta_{i}$, then the Seidel energy is expressed as the absolute sum of eigenvalues and written as

$$
S E(Z)=\sum_{i=1}^{n}\left|\theta_{i}\right|
$$

Liu Jian-ping and Liu Bo-lian have explained [14] the seidel energy. Seidel energy and its bounds have been calculated by a sharp method which is made by P. Nageswari and P. B. Sarasija [11].

Now, we discuss on Laplacian energy As $D(Z)$ is a diagonal matrix whose entries are the degree of the vertices of $Z$ and $A(Z)$ is the adjacency matrix, then the Laplacian matrix is stated as $L(Z)=D(Z)-A(Z)$. If the eigenvalues of the Laplacian matrix is denoted by $\mu_{i}$, then the Laplacian energy of the graph $Z$ is expressed as

$$
L E(Z)=\sum_{i=1}^{n}\left|\mu_{i}-d(Z)\right|
$$

where $d(Z)=2|E(Z)| /|V(Z)|$. The Laplacian matrix has been introduced by Grone and Merris [23] such as $L(Z)=D(Z)-A(Z)$. Gutman and Zhou, in 2006 [6], defined a result that energy of graph cannot be exceeded from the Laplacian energy of that graph; also, they explained some properties of Laplacian energy in [24]. Zhou has been working on energy and Laplacian energy [25] and gave some useful results. Dragan Stevanovica, Ivan Stankovicb, and Marko Milosevicb have explained some positive and negative results between the relation of energy and Laplacian energy.

We discuss some important results that are made on the path graphs by defining its spectrum, whereas the spectrum of $Z$ is the nonincreasing sequence of the distinct eigenvalues $\mu_{1}, \mu_{2}, \mu_{3}, \ldots, \mu_{n}$ of the adjacency matrix $A(Z)$ of $Z$. In particular, we explained some results regarding Randic, seidel, and Laplacian energies of NEPS of path graphs. Some results of the Randic, seidel, and Laplacian energies depend on $j$ and $i$, for $i>j$. Whereas, $j$ and $i$ denote the number of zeroes in base elements $\left(\beta_{1}, \beta_{2}, \beta_{3}, \ldots, \beta_{K}\right) \in \mathscr{B}$ and number of copies of the path graph, respectively. Also, the dimensions of the base elements are based on $i$.

Such as, if we take $j=1$ and $i=3$, then, in this case, possible base elements are

$\{(1,1,0),(1,0,1),(0,1,1)\}$. One more important point is that we will discuss on all base elements but will not includ the ones such as $(1,1, \ldots, 1)$.

\section{Main Results}

Our main focus is to compute Randic, seidel, and Laplacian energy. The broad generation of energies that is consisted on different graph matrices was the first to categorize the Laplacian energy. This is defined in the form of spectrum. The spectrum of the Laplacian matrix consists of the eigenvalues.
Theorem 1. Let $n$ and $m$ be the number of vertices and edges of NEPS $\left\{Z_{i}=P_{2_{i}}: \mathscr{B}\right\}$, respectively, for $i=2,3, \ldots, K$, where $i$ denotes the number of copies of $P_{2}$. Then,

$$
R E\left[\operatorname{NEPS}\left\{Z_{i}=P_{2_{i}}: \mathscr{B}\right\}\right]=2 m \text {. }
$$

Proof. Let $Z=\operatorname{NEPS}\left\{Z_{i}=P_{2_{i}}: \mathscr{B}\right\}$, for $i=2,3, \ldots, K$, be a graph. Here, $i$ denotes the number of copies of $P_{2}$ (which is a path graph heaving 2 vertices and 1 edge) and $\mathscr{B}$ denotes the base element which depend on the number of copies of $P_{2}$.

In that case, the spectrum of the Randic matrix of $Z$ is defined as

$$
\begin{aligned}
& \left\{\frac{2 m}{n} \quad\left(\frac{n}{2} \text { time }\right), \frac{-2 m}{n}\left(\frac{n}{2} \text { time }\right)\right\}, \\
& R E(Z)=\frac{n}{2} \times\left|\frac{2 m}{n}\right|+\frac{n}{2} \times\left|\frac{-2 m}{n}\right|=m+m=2 m .
\end{aligned}
$$

Thus, we conclude the proof:

$$
R E\left[\operatorname{NEPS}\left\{Z_{i}=P_{2_{i}}: \mathscr{B}\right\}\right]=2 m \text {. }
$$

Theorem 2. Let $n$ and $m$ be the number of vertices and edges of NEPS $\left\{Z_{i}=P_{3_{i}}: \mathscr{B}\right\}$, respectively, where number of copies of $P_{3}$ and number of zeroes in the base element are designated by $i$ and $j$, respectively. Then, for any $j$ and $i>j$, we have

$$
R E\left[\operatorname{NEPS}\left\{Z_{i}=P_{3_{i}}: \mathscr{B}\right\}\right]=3^{j} 2^{i-j} \text {. }
$$

Proof. Let $Z=\operatorname{NEPS}\left\{Z_{i}=P_{3_{i}}: \mathscr{B}\right\}$, for $i=2,3, \ldots, K$, be a graph. For any $j$ and $i>j$, we have the spectrum of the Randic matrix of $Z$ :

$$
\left\{1\left(3^{j} 2^{i-j-1} \text { time }\right), 0\left(n-3^{j} 2^{i-j} \text { time }\right),-1\left(3^{j} 2^{i-j-1} \text { time }\right)\right\} \text {. }
$$

Thus, we follow the result:

$$
\begin{aligned}
R E(Z) & =\left(3^{j} 2^{i-j-1}\right)|1|+\left(n-3^{j} 2^{i-j}\right)|0|+\left(3^{j} 2^{i-j-1}\right)|-1| \\
& =3^{j} 2^{i-j-1}+3^{j} 2^{i-j-1}, \\
R E(Z) & =R E\left[\operatorname{NEPS}\left\{Z_{i}=P_{3_{i}}: \mathscr{B}\right\}\right]=3^{j} 2^{i-j} .
\end{aligned}
$$

Theorem 3. Let $n=4^{i}$ and $m$ be the number of vertices and edges of NEPS $\left\{Z_{i}=P_{4_{i}}: \mathscr{B}\right\}$, respectively. Then, for any $j$ and $i>j$, we have

$$
R E\left[\operatorname{NEPS}\left\{Z_{i}=P_{4_{i}}: \mathscr{B}\right\}\right]=\sum_{t=0}^{i-j}{ }^{i-j} C_{t} \times 2^{i+j-t} .
$$

Proof. Let $Z=\operatorname{NEPS}\left\{Z_{i}=P_{4_{i}}: \mathscr{B}\right\}$ be a graph. Then, for any $j \geq 1$ and $i>j$, we have the spectrum of the Randic matrix as given below: 
$\left\{\frac{-1}{2^{t}}\left({ }^{i-j} C_{t} \times \frac{n}{2^{i-j+1}}\right.\right.$ time $), \frac{1}{2^{t}}\left({ }^{i-j} C_{t} \times \frac{n}{2^{i-j+1}}\right.$ time $\left.)\right\}$,

where $0 \leq t \leq i-j$,

$$
\begin{aligned}
R E(Z)= & \left(\sum_{t=0}^{i-j}{ }^{i-j} C_{t} \times \frac{n}{2^{i-j+1}}\right) \times\left|\frac{-1}{2^{t}}\right|+\left(\sum_{t=0}^{i-j}{ }^{i-j} C_{t} \times \frac{n}{2^{i-j+1}}\right) \\
& \times\left|\frac{1}{2^{t}}\right| \\
= & 2 \times\left(\sum_{t=0}^{i-j} C_{t} \times \frac{n}{2^{i-j+1}}\right) \times\left(\frac{1}{2^{t}}\right) .
\end{aligned}
$$

For $n=4^{i}$, we have

$$
R E(Z)=R E\left[\operatorname{NEPS}\left\{Z_{i}=P_{4_{i}}: \mathscr{B}\right\}\right]=\sum_{t=0}^{i-j}{ }^{i-j} C_{t} \times 2^{i+j-t} .
$$

Hence, it is done.

Theorem 4. For number of vertices and $m=n / 2$ number of edges, we have

$$
S E\left[\operatorname{NEPS}\left\{G_{i}=P_{2_{i}}: \mathscr{B}\right\}\right]=a_{\log _{2}^{n}-1}+(2 n-3),
$$

where $a_{\log _{2}^{n}-1}=a_{\log _{2}^{n}-2}+2^{\log _{2}^{n}-1}$, for $a_{1}=1$.

Proof. Let $Z=\operatorname{NEPS}\left\{Z_{i}=P_{2_{i}}: \mathscr{B}\right\}$, for $i=2,3, \ldots, K$, be a graph. Then, for $n$ vertices of $Z$, we have $m=n / 2$ edges in $Z$; we have the spectrum of the seidel matrix as

$$
\left\{a_{\log _{2}^{n}-1}, \frac{2 m}{n}\left(\frac{n}{2} \text { time }\right), \frac{-6 m}{n}\left(\frac{n}{2}-1 \text { time }\right)\right\},
$$

where $a_{\log _{2}^{n}-1}=a_{\log _{2}^{n}-2}+2^{\log _{2}^{n}-1}$, for $a_{1}=1$.

Thus, we have

$$
\begin{aligned}
\operatorname{SE}(Z) & =a_{\log _{2}^{n}-1}+\frac{n}{2}\left|\frac{2 m}{n}\right|+\left(\frac{n}{2}-1\right)\left|\frac{-6 m}{n}\right| \\
& =a_{\log _{2}^{n}-1}+4 m-\frac{6 m}{n} \\
& =a_{\log _{2}^{n}-1}+2 m\left(2-\frac{3}{n}\right)
\end{aligned}
$$

for $m=n / 2$; we have

$$
=a_{\log _{2}^{n}-1}+(2 n-3) .
$$

Theorem 5. Let $n=3^{i}$ be the number of vertices; we have $m=8 n / 9$ as edges of NEPS $\left\{Z_{i}=P_{3_{i}}: \mathscr{B}\right\}$, where $i$ is the number of copies of $P_{3}$. Furthermore, suppose that $j$ is the number of zeroes in the base element; then, for any $j \geq 1$ and $i=j+2$, we have

$$
S E\left[\operatorname{NEPS}\left\{Z_{i}=P_{3_{i}}: \mathscr{B}\right\}\right]=8 a_{j+1}+\frac{14 n-18}{9},
$$

where $a_{1}=1$ and $a_{j+1}=3 a_{j}+2$.

Proof. Let $Z=\operatorname{NEPS}\left\{Z_{i}=P_{3_{i}}: \mathscr{B}\right\}$ be the graph. Then, for $j \geq 1$ and $i=j+2$, we have the spectrum of the seidel matrix of $Z$ as given below:

$$
\left\{\frac{-45 m}{8 n}\left(a_{j+1}=3 a_{j}+2 \text { time }\right), \frac{-9 m}{8 n}\left(\frac{15 n}{27} \text { time }\right), \frac{27 m}{8 n}\right.
$$

$\left(a_{j+1}=3 a_{j}+2\right.$ time $), n-2($ sum of two eigenvalues $\left.)\right\}$,

where $a_{1}=1$ and $a_{j+1}=3 a_{j}+2$, for any $j \geq 1$.

By using this spectrum, we have

$$
\begin{aligned}
S E(Z)= & \left(a_{j+1}\right)\left|\frac{-45 m}{8 n}\right|+\left(\frac{15 n}{27}\right)\left|\frac{-9 m}{8 n}\right| \\
& +\left(a_{j+1}\right)\left|\frac{27 m}{8 n}\right|+(n-2) \\
= & \left(a_{j+1}\right)\left(\frac{45 m}{8 n}\right)+\left(\frac{15 n}{27}\right)\left(\frac{-9 m}{8 n}\right) \\
& +\left(a_{j+1}\right)\left(\frac{27 m}{8 n}\right)+(n-2) \\
= & \left(\frac{9 m}{n}\right) a_{j+1}+\frac{5 m}{8}+n-2 .
\end{aligned}
$$

For $m=8 n / 9$, we have

$$
\operatorname{SE}(Z)=\operatorname{SE}\left[\operatorname{NEPS}\left\{Z_{i}=P_{3_{i}}: \mathscr{B}\right\}\right]=8\left(a_{j+1}\right)+\frac{14 n-18}{9} \text {. }
$$

Theorem 6. Let $n$ and $m=n / 2$ be the number of vertices and edges of the graph $Z=\operatorname{NEPS}\left\{Z_{i}=P_{2_{i}}: \mathscr{B}\right\}$, respectively, for $i=2,3, \ldots, K$. Then,

$$
\operatorname{LE}\left[\operatorname{NEPS}\left\{Z_{i}=P_{2_{i}}: \mathscr{B}\right\}\right]=n \text {. }
$$

Proof. We have $n$ and $m=n / 2$ be the number of vertices and edges of the graph $Z=\operatorname{NEPS}\left\{Z_{i}=P_{2_{i}}: \mathscr{B}\right\}$, respectively, for $i=2,3, \ldots, K$. Then, the average degree of $Z$ is $d(Z)=2 m / n$.

Now, the spectrum of the Laplacian matrix can be expressed as

$$
\left\{2-\frac{2 m}{n}\left(\frac{n}{2} \text { time }\right), 0-\frac{2 m}{n}\left(\frac{n}{2} \text { time }\right)\right\} .
$$

By using this spectrum, we get the required result:

$$
L E(Z)=\frac{n}{2} \times\left|2-\frac{2 m}{n}\right|+\frac{n}{2} \times\left|0-\frac{2 m}{n}\right|=n-m+m=n .
$$

Theorem 7. Let $n$ and $m$ be the number of vertices and edges of NEPS $\left\{Z_{i}=P_{3_{i}}: \mathscr{B}\right\}$, respectively, where number of copies 
of $P_{3}$ and number of zeroes in the base element are designated by $i$ and $j$, respectively. Then, for $j \geq 1$, we have the following.

Case I: for $i=j+1$, we have

$$
L E\left[\operatorname{NEPS}\left\{Z_{i}=P_{3_{i}}: \mathscr{B}\right\}\right]=\frac{10 n}{9} .
$$

Case II: for $i=j+2$, we have

$$
\operatorname{LE}\left[\operatorname{NEPS}\left\{Z_{i}=P_{3_{i}}: \mathscr{B}\right\}\right]=\frac{106 n}{81} .
$$

Case III: for $i=j+3$, we have

$$
L E\left[\operatorname{NEPS}\left\{Z_{i}=P_{3_{i}}: \mathscr{B}\right\}\right]=\frac{1210 n}{729} \text {. }
$$

Proof. Let $n$ and $m$ be the number of vertices and edges of $Z=\operatorname{NEPS}\left\{Z_{i}=P_{3_{i}}: \mathscr{B}\right\}$, respectively, whose average degree is $2 \mathrm{~m} / \mathrm{n}$, where the number of copies of $P_{3}$ and number of zeroes in the base element are designated by $i$ and $j$, respectively.

Case I: for any $j$ and $i=j+1$, then the spectrum of the Laplacian matrix is

$$
\begin{aligned}
& \left\{3-\frac{2 m}{n}\left(\frac{n}{3} \text { time }\right), 1-\frac{2 m}{n}\left(\frac{n}{3} \text { time }\right), 0-\frac{2 m}{n}\left(\frac{n}{3} \text { time }\right)\right\}, \\
L E(Z)= & \frac{n}{3} \times\left|3-\frac{2 m}{n}\right|+\frac{n}{3} \times\left|1-\frac{2 m}{n}\right|+\frac{n}{3} \times\left|0-\frac{2 m}{n}\right| \\
= & \frac{n}{3} \times\left(3-\frac{2 m}{n}\right)+\frac{n}{3} \times\left(\frac{2 m}{n}-1\right)+\frac{n}{3} \times\left(\frac{2 m}{n}\right) \\
= & \frac{2}{3}(n+m) .
\end{aligned}
$$

In this case, for $n=3^{i}$, number of vertices, we have $m=$ $2 n / 3$ edges. Using this condition, we follow the result:

$$
\operatorname{LE}(Z)=\operatorname{LE}\left[N E P S\left\{Z_{i}=P_{3_{i}}: B\right\}\right]=\frac{10 n}{9} .
$$

Case II: for any $j$ and $i=j+2$, we have the spectrum of the Laplacian matrix as given below:

$$
\left\{5-\frac{2 m}{n}\left(\frac{n}{9} \text { time }\right), 4-\frac{2 m}{n}\left(\frac{n}{9} \text { time }\right), 2-\frac{2 m}{n}\left(\frac{2 n}{9} \text { time }\right), 1-\frac{2 m}{n}\left(\frac{n}{3} \text { time }\right), 0-\frac{2 m}{n}\left(\frac{2 n}{9} \text { time }\right)\right\} .
$$

By following this spectrum, we have calculated the Laplacian energy:

$$
\begin{aligned}
& L E(Z)= \frac{n}{9} \times\left|5-\frac{2 m}{n}\right|+\frac{n}{9} \times\left|4-\frac{2 m}{n}\right|+\frac{2 n}{9} \times\left|2-\frac{2 m}{n}\right|+\frac{n}{3} \times\left|1-\frac{2 m}{n}\right| \\
&+\frac{2 n}{9} \times\left|0-\frac{2 m}{n}\right| \\
&= \frac{n}{9} \times\left(5-\frac{2 m}{n}\right)+\frac{n}{9} \times\left(4-\frac{2 m}{n}\right)+\frac{2 n}{9} \times\left(2-\frac{2 m}{n}\right)+\frac{n}{3} \times\left(\frac{2 m}{n}-1\right)+\frac{2 n}{9} \times\left(\frac{2 m}{n}\right) . \\
& L E(Z)=\frac{2}{9}(5 n+m) .
\end{aligned}
$$

After some simplification, we obtain 
In this case, for $n=3^{i}$, number of vertices, we have $m=$ $8 n / 9$ edges. The condition has carried out this case:

$$
L E(Z)=L E\left[\operatorname{NEPS}\left\{Z_{i}=P_{3_{i}}: \mathscr{B}\right\}\right]=\frac{106 n}{81} .
$$

$$
\begin{aligned}
& \left\{9-\frac{2 m}{n}\left(\frac{n}{27} \text { time }\right), 6-\frac{2 m}{n}\left(\frac{n}{9} \text { time }\right), 4-\frac{2 m}{n}\left(\frac{n}{9} \text { time }\right),\right. \\
& \left.2-\frac{2 m}{n}\left(\frac{n}{3} \text { time }\right), 1-\frac{2 m}{n}\left(\frac{7 n}{27} \text { time }\right), 0-\frac{2 m}{n}\left(\frac{4 n}{27} \text { time }\right)\right\} .
\end{aligned}
$$

Using this spectrum, we have

$$
\begin{aligned}
L E(Z)= & \frac{n}{27} \times\left|9-\frac{2 m}{n}\right|+\frac{n}{9} \times\left|6-\frac{2 m}{n}\right|+\frac{n}{9} \times\left|4-\frac{2 m}{n}\right|+\frac{n}{3} \times\left|2-\frac{2 m}{n}\right| \\
& +\frac{7 n}{27} \times\left|1-\frac{2 m}{n}\right|+\frac{4 n}{27} \times\left|0-\frac{2 m}{n}\right| \\
= & \frac{n}{27} \times\left(9-\frac{2 m}{n}\right)+\frac{n}{9} \times\left(6-\frac{2 m}{n}\right)+\frac{n}{9} \times\left(4-\frac{2 m}{n}\right)+\frac{n}{3} \times\left(\frac{2 m}{n}-2\right) \\
& +\frac{7 n}{27} \times\left(\frac{2 m}{n}-1\right)+\frac{4 n}{27} \times\left(\frac{2 m}{n}\right) .
\end{aligned}
$$

After some calculations, we obtain

$$
L E(Z)=\frac{2}{27}(7 n+13 m)
$$

In this case, for $n=3^{i}$, number of vertices, we have $m=$ $32 n / 27$ edges. By this condition, we have

$$
L E(Z)=L E\left[\operatorname{NEPS}\left\{Z_{i}=P_{3_{i}}: \mathscr{B}\right\}\right]=\frac{1210 n}{729} .
$$

Theorem 8. Let $n=4^{i}$ and $m$ are the number of vertices and edges of NEPS $\left\{Z_{i}=P_{4_{i}}: \mathscr{B}\right\}$, respectively; then, for $j \geq 1$, we have the following.

Case I: for $i=j+1$, we have
Case II: for $i=j+2$, we have

$$
\operatorname{LE}\left[\operatorname{NEPS}\left[Z_{i}=P_{4_{i}}: \mathscr{B}\right]\right]=n \times 15731 \times 10^{-4} .
$$

Proof. Let $Z=\operatorname{NEPS}\left\{Z_{i}=P_{4_{i}}: \mathscr{B}\right\}$ be a graph that depends on $j$ and $i$. We discuss the cases for different conditions of $i$ and $j$.

Case I: for $j \geq 1$ and $i=j+1$, we have the spectrum for the Laplacian energy as

$$
\left\{0-\frac{2 m}{n}\left(\frac{n}{4} \text { time }\right), 0.5858-\frac{2 m}{n}\left(\frac{n}{4} \text { time }\right), 2-\frac{2 m}{n}\left(\frac{n}{4} \text { time }\right), 3.4142-\frac{2 m}{n}\left(\frac{n}{4} \text { time }\right)\right\},
$$

where $d(Z)=2 m / n$ is the degree of the graph, and in this case, we have $m=3 n / 4$ edges in graph $Z$ : 


$$
\begin{aligned}
L E(Z) & =\frac{n}{4} \times\left|0-\frac{2 m}{n}\right|+\frac{n}{4} \times\left|0.5858-\frac{2 m}{n}\right|+\frac{n}{4} \times\left|2-\frac{2 m}{n}\right|+\frac{n}{4} \times\left|3.4142-\frac{2 m}{n}\right| \\
& =\frac{n}{4} \times\left(\frac{2 m}{n}\right)+\frac{n}{4} \times\left(\frac{2 m}{n}-0.5858\right)+\frac{n}{4} \times\left(2-\frac{2 m}{n}\right)+\frac{n}{4} \times\left(3.4142-\frac{2 m}{n}\right) .
\end{aligned}
$$

After the simplification, we obtain

$$
L E(Z)=L E\left[\operatorname{NEPS}\left\{Z_{i}=P_{4_{i}}: \mathscr{B}\right\}\right]=n \times 12071 \times 10^{-4} .
$$

Case II: for $j \geq 1$ and $i=j+2$, we have the spectrum for the Laplacian energy as

$$
\begin{aligned}
& \left\{0-\frac{2 m}{n}\left(\frac{n}{8} \text { time }\right), 0.7076-\frac{2 m}{n}\left(\frac{n}{8} \text { time }\right), 1-\frac{2 m}{n}\left(\frac{n}{4} \text { time }\right),\right. \\
& 2.3973-\frac{2 m}{n}\left(\frac{n}{8} \text { time }\right), 3-\frac{2 m}{n}\left(\frac{n}{8} \text { time }\right) 4-\frac{2 m}{n}\left(\frac{n}{8} \text { time }\right), \\
& \left.5.8951-\frac{2 m}{n}\left(\frac{n}{8} \text { time }\right)\right\} .
\end{aligned}
$$

Now, in this case, we have $m=9 n / 8$ edges in graph $Z$ :

$$
\begin{aligned}
L E(Z)= & \frac{n}{8} \times\left|0-\frac{2 m}{n}\right|+\frac{n}{8} \times\left|0.7076-\frac{2 m}{n}\right|+\frac{n}{4} \times\left|1-\frac{2 m}{n}\right|+\frac{n}{8} \times\left|2.3973-\frac{2 m}{n}\right| \\
& +\frac{n}{8} \times\left|3-\frac{2 m}{n}\right|+\frac{n}{8} \times\left|4-\frac{2 m}{n}\right|+\frac{n}{8} \times\left|5.8951-\frac{2 m}{n}\right| \\
= & \frac{n}{8} \times\left(0-\frac{2 m}{n}\right)+\frac{n}{8} \times\left(0.7076-\frac{2 m}{n}\right)+\frac{n}{4} \times\left(1-\frac{2 m}{n}\right)+\frac{n}{8} \times\left(2.3973-\frac{2 m}{n}\right) \\
& +\frac{n}{8} \times\left(3-\frac{2 m}{n}\right)+\frac{n}{8} \times\left(4-\frac{2 m}{n}\right)+\frac{n}{8} \times\left(5.8951-\frac{2 m}{n}\right) .
\end{aligned}
$$

After some calculations, we get the result

$$
L E(Z)=L E\left[\operatorname{NEPS}\left\{Z_{i}=P_{4_{i}}: \mathscr{B}\right\}\right]=n \times 15731 \times 10^{-4} \text {. }
$$

\section{Conclusion}

In this study, we investigated the Randic, seidel, and Laplacian energies of the NEPS of path graph $P_{n_{i}}$ with any base $\mathscr{B}$. Here, $n$ denotes the number of vertices and $i$ denotes the number of copies of path graph $P_{n}$. Some of the results depend on the number of zeroes in base elements, for which we use the notation $j$.

\section{Data Availability}

The data used to support the work are cited within the article as references.

\section{Conflicts of Interest}

The authors declare that they have no conflicts of interest.

\section{Authors' Contributions}

All authors contributed equally to this work.

\section{References}

[1] D. Cvetkovic and S. Simic, "Non-complete extended p-sum of graphs, graph angles and star partitions," Publications de l'Institut Mathématique, vol. 53, no. 67, pp. 4-16, 1993.

[2] D. Cvetkovic and M. Petric, "Connectedness of the noncomplete extended p-sum of graphs," Zb. Rad., Prir.-Mat. Fak., Univ. Novom Sadu, Ser. Mat, vol. 13, pp. 345-352, 1983.

[3] D. M. Cvetković and R. P. Lučić, "A new generalization of the concept of the p-sum of graphs. Publikacije Elektrotehničkog fakulteta," Serija Matematika i fizika, vol. 18, pp. 67-71, 1970 . 
[4] K. C. Das, S. Sorgun, and K. Xu, "On Randic energy of graphs," MATCH Communications in Mathematical and in Computer Chemistry, vol. 72, no. 1, pp. 227-238, 2014.

[5] I. Gutman, "The energy of a graph," Ber. Math. Stat. Sekt. Forsch. Graz, vol. 103, pp. 1-22, 1978.

[6] I. Gutman and B. Zhou, "Laplacian energy of a graph," Linear Algebra and Its Applications, vol. 414, no. 1, pp. 29-37, 2006.

[7] W. H. Haemers, "Seidel switching and graph energy," MATCH Communications in Mathematical and in Computer, vol. 68, pp. 653-659, 2012.

[8] W. W. Kirchherr, "NEPS operations on cordial graphs," Discrete Mathematics, vol. 115, no. 1-3, pp. 201-209, 1993.

[9] I. Gutman and B. Furtula, "Recent results in the theory of randic index," University of Kragujevac, Kragujevac, vol. 14, pp. 22-35, 2008.

[10] I. Gutman, B. Furtula, and Ş. B. Bozkurt, "On Randić energy," Linear Algebra and Its Applications, vol. 442, pp. 50-57, 2014.

[11] P. Nageswari and P. B. Sarasija, "Seidel energy and its bounds," International Journal of Mathematics and Analysis, vol. 8, pp. 2869-2871, 2014.

[12] M. R. Oboudi, "Energy and Seidel energy of graphs," MATCH Communications in Mathematical and in Computer, vol. 75, pp. 291-303, 2016.

[13] D. Stevanović, "Energy and NEPS of graphs," Linear and Multilinear Algebra, vol. 53, no. 1, pp. 67-74, 2005.

[14] J.-p. Liu and B.-1. Liu, "Generalization for laplacian energy," Applied Mathematics-A Journal of Chinese Universities, vol. 24, no. 4, pp. 443-450, 2009.

[15] X. Li and I. Gutman, Mathematical Aspects of Randic-type Molecular Structure Descriptors, University of Kragujevac, Kragujevac, Serbia, 2006.

[16] X. Li and Y. Shi, "A survey on the Randic index," MATCH Communications in Mathematical and in Computer, vol. 59, pp. 127-156, 2008.

[17] J.-B. Liu, J. Zhao, and Z. Cai, "On the generalized adjacency, Laplacian and signless Laplacian spectra of the weighted edge corona networks," Physica A, vol. 540, pp. 123-133, 2020.

[18] J.-B. Liu and X.-F. Pan, "Asymptotic incidence energy of lattices," Physica A: Statistical Mechanics and Its Applications, vol. 422, pp. 193-202, 2015.

[19] J.-B. Liu and X.-F. Pan, "Asymptotic Laplacian-energy-like invariant of lattices," Applied Mathematics and Computation, vol. 253, pp. 205-214, 2015.

[20] S. C. Shee, "A note on the C-product of graphs," Nanta Math, vol. 7, no. 2, pp. 105-108, 1974.

[21] M. Randic, "On history of the Randic index and emerging hostility toward chemical graph theory," MATCH Communications in Mathematical and in Computer, vol. 59, pp. 51-64, 2008.

[22] M. Randic, "On characterization of molecular branching," Journal of the American Chemical Society, vol. 97, pp. 66096615, 1975.

[23] R. Grone and R. Merris, "The Laplacian spectrum of a graph II," SIAM Journal on Discrete Mathematics, vol. 7, no. 2, pp. 221-229, 1994.

[24] H. Wang and H. Hua, "Note on Laplacian energy of graphs," MATCH Communications in Mathematical and in Computer, vol. 59, no. 2, pp. 373-380, 2008.

[25] B. Zhou, "More on energy and Laplacian energy," MATCH Communications in Mathematical and in Computer, vol. 64, no. 1, pp. 75-84, 2010. 